\title{
VirTual MACHINe SCHEDUling IN ClOUd COMPUTING ENVIRONMENT
}

\author{
Yousef Tohidirad $^{1}$, Siamak Abdezadeh ${ }^{2}$, Zahed Soltani aliabadi $^{3}$, Abdolsalam $^{-}$ \\ Azizi $^{4}$ and Mohammad Moradi ${ }^{5}$ \\ ${ }^{1}$ Department of Computer Engineering, Electronic Branch, Islamic Azad University, \\ Tehran, Iran \\ ${ }^{2}$ Department of Computer, Boukan Branch, Islamic Azad University, Boukan, Iran \\ ${ }^{3,4}$ Department of Computer Engineering, Urmia Branch, Islamic Azad University, Urmia, \\ Iran \\ ${ }^{5}$ Department of Computer, baneh Branch, Islamic Azad University, baneh, Iran
}

\begin{abstract}
Cloud computing is an upcoming technology in dispersed computing facilitating paying for each model as for each user demand and need. Cloud incorporates a set of virtual machine which comprises both storage and computational facility. The fundamental goal of cloud computing is to offer effective access to isolated and geographically circulated resources. Cloud is growing every day and experiences numerous problems such as scheduling. Scheduling means a collection of policies to regulate the order of task to be executed by a computer system. An excellent scheduler derives its scheduling plan in accordance with the type of work and the varying environment. This research paper demonstrates a generalized precedence algorithm for effective performance of work and contrast with Round Robin and FCFS Scheduling. Algorithm needs to be tested within CloudSim toolkit and outcome illustrates that it provide good presentation compared some customary scheduling algorithm.
\end{abstract}

\section{KEYWORDS}

CloudSim, Virtual Machine, Cloud Computing, Scheduling, FCFS Scheduling

\section{INTRODUCTION}

To Use cloud computing technology users need just to take a regular PC, high speed internet connection and a good browser and connect to their cloud. Two main reasons for using cloud computing is to maximize performance and minimize costs $[1,2]$. Cloud computing reduce heavy hardware costs for companies. For example, we don't need to buy a high capacity hard disks and advanced processors. Furthermore, there is no need for physical storage space but only pay for rent and put the information on the store tool and access our data. A cloud computing system is also faster boot and setup because in that case computers have fewer programs and process that will load into memory. The performance of this computer compared with other computing systems is optimized with maximum performance [1,3]. One of these methods to reduce the overall cost of server consolidation is virtualization $[1,4]$ which is the most widely used method in cloud computing as a cloud computing infrastructure [1]. Cloud computing focuses on creation of virtualization, grid computing and web technologies. It is defined as internet centered computing that provides software as service SaaS, platform as service PaaS, and infrastructure as a service (ISaaS). Software app is made accessible by the cloud provider in SaaS. In IaaS the computing infrastructure is offered as a service to the client in the shape of Virtual Machine (VM). In PaaS an app creation platform is offered as a service to the developer to generate a web based app [5]. All these services are made accessible on contribution basis through pay-as-you-

DOI : 10.5121/ijmpict.2015.6401 
International Journal of Managing Public Sector Information and Communication Technologies (IJMPICT), Vol. 6, No. 4, December 2015

use model to clients, in spite of their locality. Cloud computing is still under development stage and characterize several concerns out of different challengers in cloud scheduling plays incredibly vital role in identifying the efficient implementation [6].

Scheduling means to establish policies to regulate the sequence of task to be executed using a processor system. There have been different forms of scheduling algorithm available in the dispersed computing system, and work scheduling is part on them. The key benefit of job scheduling algorithm is to attain a great execution computing and the most excellent system all through. Scheduling similarly manages the existing computer memory and better scheduling plan provides utmost application of resource [7].

Including important features for scheduling algorithms are distribution and planning of resources, in section two the joint approach and framework to offer them. In the third section of the article, mentioned Methods of planning algorithms and the simulation algorithm. Also in In the third section, Is proposed overall prioritization algorithm for better performance and compare it with the FCFS scheduling algorithm and Clearly presented and compared the effective performance of the Prioritization algorithm against algorithm listed is shown. Conclusions will be discussed in section Four.

\section{Proposed FAMe Work ANd Methodology}

Resource distribution and scheduling of resources remains significant feature that impacts the concert of a networking, analogous cloud computing and distributed computing [8]. Numerous researchers have suggested a variety of algorithms for distributing, scaling and scheduling the resources effectively in a cloud scheduling course can be produced into stages that is to say:

- Resource determining and filtering - Datacenter agent identifies resources available in within the network and gathers status data associated to them.

- Resource assortment - aim resources are chosen based on particular factors of resource and task. This is referred as deciding stage [5].

- Task submission - in this stage, job is submitted to the selected source.

According to this description, Tabel 1 and figure 1 shows the Result comparison of available algorithms in Different datacenters.

TABLE 1. Result comparison of available algorithms [9]

\begin{tabular}{|l|c|c|c|}
\hline Algorithms & Datacenter_0 & Datacenter_1 & Datacenter_2 \\
\hline $\begin{array}{l}\text { Algorithm to select host } \\
\text { with minimum PE's }\end{array}$ & $7179.2 \mathrm{debt}$ & $7179.2 \mathrm{debt}$ & $1025.6 \mathrm{debt}$ \\
\hline $\begin{array}{l}\text { Dynamic VM allocation } \\
\text { with Clustering }\end{array}$ & $5128 \mathrm{debt}$ & $5128 \mathrm{debt}$ & $3076.8 \mathrm{debt}$ \\
\hline
\end{tabular}




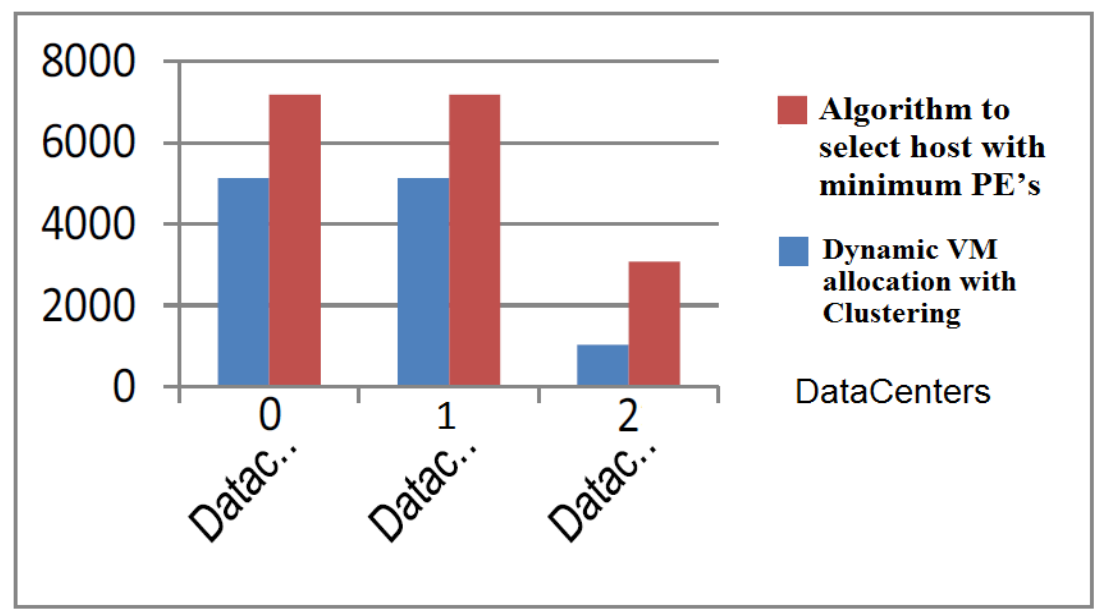

Figure 1. Performance comparison of the Proposed Algorithm having allocation Algorithm by similar input parameter [9]

\section{Methods OF Algorithm SCHEDULING}

A) First come first serve (FCFS): It is for parallel processing and targets the resource having the least waiting line up time and is chosen for the received job. The Sim toolkit backs FCFS scheduling plan for interior scheduling tasks. Distribution of app-specified VMs to Hosts within a Cloud-based datacenter is the work of the virtual machine stipulated element [7]. The default policy adopted by the VM stipulated is a simple policy that distributes a VM in FCFS method. The limitations of First come first serve is that it is non preventative. The shortest errands which are based at the back of the line-up must wait for long errands at the front to complete. It is turnaround and reaction is fairly minimal [7].

B) Round Robin Scheduling (RS): RS algorithm targets on the equality. It uses the loop as it queue to store tasks. Each task within the queue has similar implementation time and it will be implemented as a result. If the task cannot be finished during its moment, it will be retained back to the line-up waiting for the succeeding turn [10]. The benefit of Round Robin algorithm is that each task will be implemented as a result and they do not have to be waited for the preceding one to be finished. But is the weight is discovered to be heavy, Round Robin will take longer to finish all the tasks. RR scheduling strategy will be supported by CloudSim toolkit for interior scheduling of tasks. The disadvantage of Round Robin is that the prime work takes sufficient period to for completion $[11,12]$.

C) Generalized Priority Algorithm: Client classify in accordance with the consumer demand. You should classify the limitation such as memory, size, bandwidth scheduling and others. It is the recommended strategy; the jobs are originally prioritized depending on their size in a way that one with the largest size has largest score $[10,11,12]$. The virtual machine is similarly prioritized in accordance to their MIPS quality in a way that one with highest MIPS ranks the highest. Therefore, the main factor for ranking jobs is their size and MIPS for VM. This policy is doing well than RR and FCFS scheduling [5]. 
International Journal of Managing Public Sector Information and Communication Technologies (IJMPICT),

Vol. 6, No. 4, December 2015

According to the method described, figure 2 shows Structure of reservation cluster-based cloud computing approach.

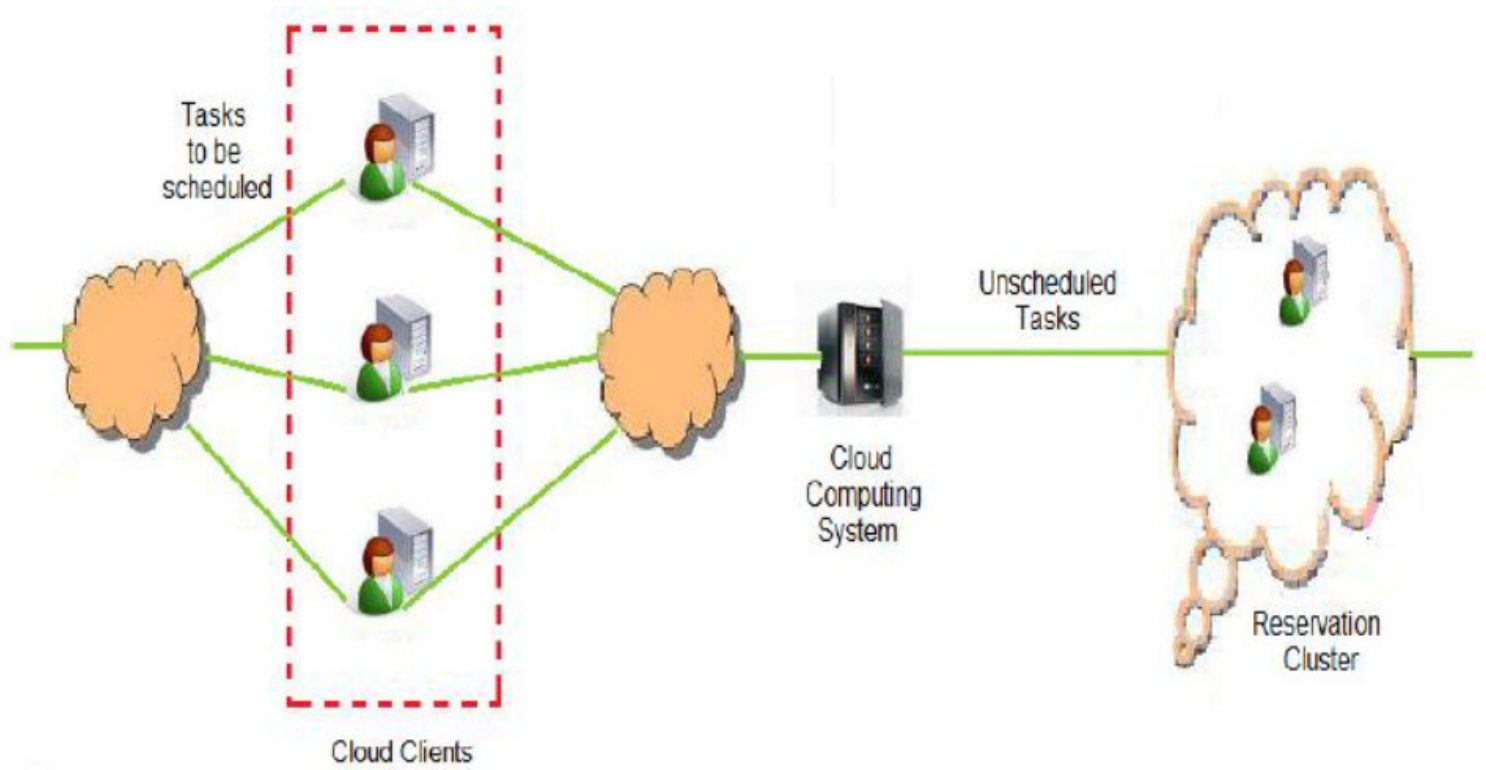

Figure 2. Structure of reservation cluster-based cloud computing approach [2]

\subsection{Algorithm Solutions}

The algorithm shown in Figure 3, stores every appropriate Virtual Machine within VM List.

\begin{tabular}{|l}
\hline Prev 99 \\
Push first vertex \\
While stck 6 = Empty do \\
Get unvisited vertex adjacent close to \\
stack top \\
If no adjacent vertex then \\
If prev = 6 StackTop then \\
Copy all stack contents to VM List \\
End if \\
Else \\
Mark the node as visited \\
Push close vertex \\
End if \\
End while
\end{tabular}

Figure 3. Storing every appropriate Virtual Machine within VM List

\subsection{Various steps of algorithm solution}

Step 1 - Generate VM to unlike Datacenter in accordance to computational strength of physical/host server in provisions of its prize CPU, CPU speed, storage and memory.

Step 2 - Distribute cloudlet size in accordance to computational strength 
International Journal of Managing Public Sector Information and Communication Technologies (IJMPICT), Vol. 6, No. 4, December 2015

Step 3 - VM weight balance retain and index table of VMs, currently VM comprise zero distribution [10].

Step 4 - Cloudlet bound in accordance to MIPS and particular length.

Step 5 - Largest length of cloudlet acquire largest MIPS of VM.

Step 6 - Datacentre agents sends the application to the virtual machine.

Step 7 - Update the obtainable resource.

According to the steps described, table 2 shows List of parameters are sorted according to memory required and table 3 Available memory after allocation of processes to virtual machines.

TABLE 2. List of parameters are sorted according to memory required [13]

\begin{tabular}{|c|c|c|c|c|c|c|}
\hline $\begin{array}{c}\text { User } \\
\text { ID }\end{array}$ & Arrival & $\begin{array}{c}\text { Process } \\
\text { Time }\end{array}$ & Deadline & $\begin{array}{c}\text { I/O } \\
\text { request }\end{array}$ & $\begin{array}{c}\text { Memory } \\
\text { request }\end{array}$ & $\begin{array}{c}\text { Type of } \\
\text { service }\end{array}$ \\
\hline 6 & 82 & 7 & 93 & 4 & 1102 & 0 \\
\hline 3 & 91 & 8 & 109 & 3 & 1143 & 1 \\
\hline 5 & 17 & 8 & 27 & 1 & 1476 & 0 \\
\hline 2 & 97 & 10 & 113 & 4 & 4541 & 0 \\
\hline 9 & 16 & 2 & 24 & 4 & 10893 & 1 \\
\hline 4 & 93 & 7 & 107 & 3 & 12554 & 1 \\
\hline 7 & 38 & 8 & 53 & 0 & 15670 & 0 \\
\hline 8 & 64 & 8 & 79 & 1 & 21749 & 1 \\
\hline 10 & 22 & 8 & 34 & 2 & 22369 & 1 \\
\hline 1 & 9 & 3 & 18 & 4 & 30875 & 0 \\
\hline
\end{tabular}

TABLE 3. Available memory after allocation of processes to virtual machines [13]

\begin{tabular}{|c|c|c|c|c|c|c|c|c|}
\hline \multirow{2}{*}{ Cloud No. } & Arrival & \multicolumn{7}{|c|}{ Virtual machine number related with cloud } \\
\cline { 2 - 9 } & 1 & 2 & 3 & 4 & 5 & 6 & 7 & 8 \\
\hline 1 & 12847 & 3776 & 10249 & 9629 & 1123 & 3200 & 3200 & 3200 \\
\hline 2 & 3200 & 3200 & 3200 & 3200 & 3200 & 3200 & 3200 & 3200 \\
\hline 3 & 3200 & 3200 & 3200 & 3200 & 3200 & 3200 & 3200 & 3200 \\
\hline 4 & 3200 & 3200 & 3200 & 3200 & 3200 & 3200 & 3200 & 3200 \\
\hline 5 & 3200 & 3200 & 3200 & 3200 & 3200 & 3200 & 3200 & 3200 \\
\hline 6 & 3200 & 3200 & 3200 & 3200 & 3200 & 3200 & 3200 & 3200 \\
\hline
\end{tabular}

\subsection{Experiment and Evaluation}

So as to confirm the algorithm, experiment on core (TM) i5 computer $2.6 \mathrm{GHz}$, Cloud Sim 3.0.3 simulator and Windows 7 platform. The Cloud Sim toolkit maintains reproduction of cloud system elements for example virtual machines, data centers, scheduling, hosts and resource provisioning strategies [7]. A tool kit is the use which opens the probability of assessing the theory before software development within an environment where you can duplicate tests available [11]. For comparison evaluation, tables are used to demonstrate implemented generalized priority algorithm, FCFS, and Round Robin methodology. 
International Journal of Managing Public Sector Information and Communication Technologies (IJMPICT), Vol. 6, No. 4, December 2015

\section{CONCLUSIONS}

Scheduling is one of the greatest vital jobs in cloud computing background. Scheduling is very much necessary to improve the server and resource utilization also to increases the performance of the computer. The different scheduling algorithm methods discussed above effectively schedules the computational jobs in cloud background. Priority remains a significant matter of task scheduling in cloud backgrounds. The experiment is conducted for unreliable number of VMs workload outlines. The experiment carried out is proportionate to Round Robin and FCFS algorithm.

\section{REFERENCES}

[1] M. Masdari, B. Zebardast, Y. Lotfi, "Towards Virtualization in Cloud Computing”, International Journal of Advanced Research in Computer Science, Vol.4, No. 4, pp.96-101, 2013.

[2] K .Vasantha Kokilam, Samson Dinakaran, "Data Storage Virtualization in Cloud Computing", International Journal of Advanced Research In Technology(IJART), Vol. 1, Issue 1, pp. 16-21, 2011.

[3] Vaquero Luis M., Rodero-Merino Luis, Caceres Juan, Lindner Maik, "A Break in the Clouds: Towards a Cloud Definition", ACM SIGCOMM Computer Communication Review, Vol.39, No.1, pp. 50-55, January 2009.

[4] A. Weiss, "Computing in the clouds", netWorker - Cloud computing: PC functions move onto the web, Vol. 11, Issue 4, pp. 16-25, December 2007.

[5] H. Salimi, "Advantages, Challenges and Optimizations of Virtual Machine Scheduling in Cloud Computing Environments", International Journal of Computer Theory and Engineering, pp.189193, 2013.

[6] M. Alam, "Cloud Algebra for Handling Unstructured Data in Cloud Database Management System”, International Journal on Cloud Computing: Services and Architecture, pp.35-42, 2012.

[7] H. Yuan, C. Li, M. Du, "Optimal Virtual Machine Resources Scheduling Based on Improved Particle Swarm Optimization in Cloud Computing”, JOURNAL OF SOFTWARE, 2014.

[8] K. D. prajapati, P. Raval, M. Karamta, M. Potdar, "Comparison of Virtual Machine Scheduling Algorithms in Cloud Computing", International Journal of Computer Applications, pp.12-14, 2013.

[9] B. Panchal, R. K. Kapoor, "Dynamic VM Allocation Algorithm using Clustering in Cloud Computing", International Journal of Advanced Research in Computer Science and Software Engineering, pp.143-150, 2013.

[10] G. Raj, "Effective Cost Mechanism for Cloudlet Retransmission and Prioritized VM Scheduling Mechanism Over Broker Virtual Machine Communication Framework", International Journal on Cloud Computing: Services and Architecture, pp.41-50, 2012.

[11] Y. Cao, C. Ro, "Adaptive Scheduling for QoS-based Virtual Machine Management in Cloud Computing”, International Journal of Contents, pp.7-11, 2012.

[12] S. Paulus, U. Riemann, "An Approach for a Business-driven Cloud-compliance Analysis Covering Public Sector Process Improvement Requirements", International Journal of Managing Public Sector Information and Communication Technologies (IJMPICT), Vol. 4, No. 3, pp. 1-13, 2013.

[13] R. Saini, Indu, "EFFICIENT JOB SCHEDULING OF VIRTUAL MACHINES IN CLOUD COMPUTING", International Journal of Advanced Research in Computer and Communication Engineering, pp.3349-3354, 2013. 